\title{
Detecting response shift in health-related quality of life measurement among patients with hypertension using structural equation modeling
}

\author{
Hao Chen ${ }^{1,3}$, Lin Zhu' ${ }^{1}$, Rui Zhou' ${ }^{1}$ Panpan Liư ${ }^{2}$, Xiaoyang Lu ${ }^{3}$, Donald L. Patrick ${ }^{4}$, Todd C. Edwards ${ }^{4}$ and \\ Hongmei Wang ${ }^{1,3^{*}}$ (i)
}

\begin{abstract}
Background: Outcomes derived from longitudinal self-reported health-related quality of life measures can be confounded by response shift. This study was aimed to detect response shift among patients with hypertension attending a community-based disease management program.

Methods: 240 consecutive consulting or follow-up patients with diagnosed hypertension were recruited. The Short Form 36-item Health Survey was self-administered at 12 community health service stations at baseline and four weeks after attending the program. The 4-step structural equation modeling approach assessed response shift.

Results: Data from 203 (84.6\%) patients were eligible for analyses (mean age 65.9 \pm 10.8 years, $46.3 \%$ female). The results showed uniform recalibration of social functioning $\left(\chi_{\text {SBdiff }}^{2}(1)=22.98, P<0.001\right)$, and non-uniform recalibration of role limitations due to physical problems $\left(\chi_{\text {SBdiff }}^{2}(1)=8.84, P=0.003\right)$, and bodily pain $\left(\chi_{\text {SBdiff }}^{2}(1)=17.41, P<0.001\right)$. The effects of response shift on social functioning were calculated as "small" (effect-size $=0.35$ ), but changed the observed changes from improvement (effect-size $=0.25$ ) to slight deterioration (effect-size $=-0.10$ ). After accounting for the response shift effect, the general physical health of participants was improved (effect-size $=0.37$ ), while deterioration (effect-size $=-0.21$ ) in the general mental health was also found.
\end{abstract}

Conclusions: Recalibration existed among patients with hypertension attending the disease management program. The interventions in the program might act as a catalyst that induced the response shift. We conclude that response shift should be considered in hypertension research with longitudinal health-related quality of life data.

Keywords: Health-related quality of life, Response shift, Structural equation modeling, Hypertension, SF-36

\section{Background}

Health-related quality of life (HRQOL), representing people's subjective assessment of their sense of healthspecific well-being, has been frequently used as a

\footnotetext{
*Correspondence: rosa@zju.edu.cn

${ }^{1}$ Department of Social Medicine of School of Public Health, Zhejiang University School of Medicine, 866 Yuhangtang Road, Hangzhou 310058, Zhejiang, People's Republic of China

Full list of author information is available at the end of the article
}

health indicator in medical interventions or health surveys. However, the measurement of change in HRQOL may be affected by the fact that individuals' frame of reference (or standard) or the concept and meaning of HRQOL can differ over time, known as Response Shift (RS) $[1,2]$. A theoretical model (Additional file 1: Figure S1) of RS have been proposed by Sprangers and Schwartz, who postulated a dynamic feedback loop, where "catalyst," "antecedents," "mechanisms" and 
"RS" interacted and eventually maintained or led to changes in HRQOL [1], among which, RS was defined as three different types: (a) recalibration: a change in the respondent's internal standards of measurement; (b) reprioritization: a change in the respondent's values; and (c) reconceptualization: a redefinition of the target construct. With the existence of RS, individual experience of improvement or deterioration over time will be modified. In other words, substantial change of HRQOL can be over-or under-estimated without adjusting for RS [3-5]. Therefore, it is important to consider RS effect when measuring changes in HRQOL [6].

A variety of methodological methods are available to detect and adjust RS [2]. Based on the latent variable measurement modeling, Oort et al. [7, 8] have proposed a 4-step RS detecting procedure for longitudinal measurement occasions, named the Oort's structural equation modeling (SEM) approach. The invariance of the corresponding parameters' magnitude or model patterns across occasions was associated with the interpretation of all three RS types. Due to its versatility, Oort's SEM approach has become the most widely used statistical method in RS detection [9].

Hypertension is a common chronic disease and a major risk factor for cardiovascular chronic diseases [10]. Many studies have confirmed that hypertension has been an influencing factor for the deterioration of HRQOL [11-14]. Previous research has provided evidence that recalibration type of RS existed among hypertensive male subjects [15] and hypertensive patients with coronary artery disease (CAD) [16]. In contrast, there is still a dearth of research that addresses RS phenomena against general hypertensive patients.

The community disease management program is a component of the national essential public health services in China, providing disease screening, drug therapy, long-term follow-up, and health education services to improve hypertension care [17]. Previous studies have found that patients with multiple chronic diseases changed perspective on their health status after attending self-management courses $[18,19]$. The disease management program interventions may act as catalysts for psychological coping mechanisms, and subsequently affect how individuals think about their quality of life, which could in turn induce RS [20].

This study's objective was to detect the effects of RS on HRQOL changes in patients with hypertension involved in the community disease management program. We hypothesized that patients who undergo this program would experience RS.

\section{Methods}

\section{Study design and samples}

The present study was a phase two study of a research project on quality of life of patients with common chronic diseases in the community health service and disease management strategy. In phase one of this project, the quality of life of consulting patients in the community health service was compared with those of the general population. Experimental studies of disease management for hypertension or diabetes patients were conducted in phase three [21-24].

The study cohort recruited 240 patients with hypertension in the community disease management program from a community health service center in Hangzhou, China. On the given week chosen by the study, all visiting or followed up patients in the belonged 12 community health service stations were invited to self-administer a health status survey before consultation until the quota for each station $(n=20)$ was met (baseline). Four weeks later, the participants were asked to complete the survey once again (follow-up). Patients were eligible if they (1) had a diagnosed hypertension ( $\mathrm{SBP} \geq 140 \mathrm{mmHg}$ and/or $\mathrm{DBP} \geq 90 \mathrm{mmHg}$ ), (2) were included in the community disease management program, and (3) had a regular follow-up. Patients with cognitive or visual problems or were unable to complete the questionnaires independently were excluded. The Ethics Committee of Zhejiang University School of Medicine approved the protocol, and all participants provided written informed consent.

\section{Measures}

We used a validated Chinese (mainland) version of the Short-Form Health Survey (SF-36) to evaluate the HRQOL of patients with hypertension in this study [25]. The SF-36 instrument consists of 36 items which measure eight scales: physical functioning $(\mathrm{PF})$, role limitations due to physical problems (RP), bodily pain $(\mathrm{BP})$, general health $(\mathrm{GH})$, vitality $(\mathrm{VT})$, social functioning (SF), role limitations due to emotional problems (RE), and mental health (MH). All original scales were linearly transformed to a scale from 0 to 100 , with a higher score indicating better HRQOL [26]. The demographic and disease information, including age, gender, marital status, employment status, educational attainment, health insurance, self-reported severe illness experience, duration of disease, blood pressure, health risk level (evaluated based on blood pressure, risk factors, target organ damage/diabetes mellitus, and multi-morbidities [17]), and medicine taken were also collected. 


\section{Analyses}

\section{Structural equation modeling}

The Oort's SEM approach was applied to detect RS in a 4-step procedure [7, 8]: (1) establishing a decent measurement model; (2) forming a no RS model; (3) detecting RS; (4) evaluating adjusted change.

Step 1: in this step, we first established a 2-factor construct model for baseline data guided by exploratory factor analyses (EFA) and the published principal components analyses of the SF-36 [26]. We further applied it at both baseline (T1) and follow-up (T2), arrived at a longitudinal model. Combined with the guidance of modification index and substantive consideration, we modified it to develop the measurement model (Model 1). In model 1 , all RS-related parameters (residual variances, intercepts, factor loadings) could be estimated freely across occasions. The common factors means and variances were constrained at zero and one respectively, as the scales and origins for the unobserved variables (i.e., common factors). Only when model 1 shows an acceptable fit could further analyses be conducted. A variety of model fit indexes were used to assess the appropriateness of model fit. These include the comparative fit index (CFI), the Tucker-Lewis index (TLI), the standardized root mean square residual (SRMR), and the root mean square error of approximation (RMSEA). With CFI, TLI $>0.9$, SRMR $<0.1$, and RMSEA $<0.08$ indicate acceptable model fit $[27,28]$.

Step 2: the invariance constraints on all RS-related parameters were placed across occasions (i.e., setting the paired parameters equal over time), forming the no RS model (Model 2). By using the $\chi^{2}$ difference test, the overall existence of RS could be detected. If the model fit of model 2 was significantly worse than model 1 , we could conclude that RS existed.

Step 3: in the third step, RS was detected by checking whether the fit of Model 2 can be improved significantly by releasing across occasion invariance constraints on RS-related parameters. Guided by the modification index, the across-occasion invariance of RS-related parameters was tested by the $\chi^{2}$ difference test step-by-step. Invariance constraints that had been proved untenable (i.e., releasing these constraints led to significant improvement in model fitting) were removed individually, leading to a model (Model 3) where no modification index of RSrelated parameters indicated a significantly better fitting (i.e., all RS was taken into account). Different types of RS were operationalized by the following parameters that varied across occasions: reconceptualization (factor patterns), reprioritization (factor loadings), uniform recalibration (intercepts), ununiform recalibration (residual variances). Given the backward approach could result in over identification of RS, a Bonferroni-adjusted critical value of $0.05 / 8$ was used to control type I error [16, 29, 30].

Step 4: as the final step, all invariance constraints of common factors means, variances, and correlations were tested by checking if they can improve the fit of model 3. Tenable constraints were placed on the final model (Model 4). In this model, the adjusted change was assessed by testing the invariant hypothesis of common factor means across occasions after accounting for all RS. The estimated parameters in model 4 were used to calculate the effect-sizes of RS and the adjusted change. Effect size values of Cohen's $d=0.2,0.5$, and 0.8 are considered "small", "medium", and "large" [31].

\section{Statistical analyses}

Descriptive statistics were used to characterize the study sample. We then applied $t$ test, one-way ANOVA, Chisquare, or Fisher's Exact tests to compare demographic, health conditions, and HRQOL scores on a subsample with baseline data only or missing data (the attrition sample) and one with eligible data (the analytic sample) to examine selection bias. These analyses were implemented using IBM SPSS version 24.

Mplus version 7.4 was used for the SEM analyses. Given our data deviated from multivariate normality, the Robust Maximum Likelihood Estimator (MLR) was employed as the estimator [32]. When conducting the $\chi^{2}$ difference tests in Mplus with the MLR estimator, it was essential to adjust the $\chi^{2}$ statistics. The Satorra-Bentler scaling correction was conducted for $\chi^{2}$ statistics adjustment [33].

\section{Results}

Participants' characteristics and health information

The initial cohort included 240 patients. 211 (87.9\%) patients completed the questionnaire twice, while 8 patients with missing data were excluded, resulting in a data set of $203(84.6 \%)$ patients used for analyses. Among 203 patients with eligible data, 94 were female (46.3\%), and the mean age was 65.9 years (range $35-86$, SD 10.8). About one fifth (19.2\%) of participants had college or above education. Four fifths $(75.4 \%)$ of participants were unemployed or retired. More than four fifths (83.7\%) of participants had an annual household income of no more than 60,000 RMB. Seven (3.3\%) participants did not have any health insurance (Table 1).

About one of the third participants reported their own or family member's serious disease experience. Sixteen $(7.8 \%)$ participants had hypertension no more than six months. More than half (54.2\%) of participants had average blood pressure lower than 140/90 $\mathrm{mmHg}$. About three fourths (74.4\%) of participants had a low or 
Table 1 Demographic characteristics of study participants

\begin{tabular}{|c|c|c|}
\hline Variable & $\mathrm{N}$ & $\mathrm{n}(\%)$ \\
\hline Gender & 203 & \\
\hline Female & & $94(46.3)$ \\
\hline Male & & $109(53.7)$ \\
\hline Marital status & 201 & \\
\hline Married/co-habiting & & $181(89.2)$ \\
\hline Other & & $20(9.6)$ \\
\hline Education attainment & 201 & \\
\hline No school & & $6(3.0)$ \\
\hline Elementary school & & $55(27.1)$ \\
\hline Middle school & & $60(29.6)$ \\
\hline High school or vocational training & & $41(20.2)$ \\
\hline College or above & & $39(19.2)$ \\
\hline Employment status & 191 & \\
\hline Employed & & $30(14.8)$ \\
\hline Not employed/retired & & $153(75.4)$ \\
\hline Full-time housework & & $8(3.9)$ \\
\hline Annual household income ( $\$ 1 \cup S=6.8 Y$ uan) & 203 & \\
\hline$<60,000 \mathrm{RMB}$ & & $170(83.7)$ \\
\hline$\geq 60,000 \mathrm{RMB}$ & & $27(13.3)$ \\
\hline Health insurance & 203 & \\
\hline Urban employee basic medical insurance & & $80(39.4)$ \\
\hline Urban resident basic medical insurance & & $107(52.7)$ \\
\hline Other insurances ${ }^{a}$ & & $9(4.3)$ \\
\hline No insurance & & $7(3.3)$ \\
\hline
\end{tabular}

a Other insurances include new cooperative medical scheme, commercial medical insurance, free medical service, etc.

medium level of health risks. Sixteen (7.9\%) participants did not take any medicine (Table 2).

\section{Selection biases}

The examination of differences between the attrition sample and analytic sample revealed that they were comparable on almost all demographic characteristics and health conditions. However, there were differences between the two samples, such that patients from the attrition sample were more likely to do full-time housework $(\mathrm{P}=0.032)$. A comparison of HRQOL scores between the two samples revealed that the attrition sample reported slightly worse physical functioning $(\mathrm{P}=0.028)$ and role limitations due to physical problems $(\mathrm{P}=0.013)$ than the analytic sample (see Additional file 2: Table S1).

\section{Structural equation modeling Measurement model}

We fixed two factors in EFA according to the principal components model of the SF-36 scales described by Ware et al. [26]. The two common factors were named as GenPHYS (General physical health) and GenMENT (General
Table 2 Health conditions of study participants

\begin{tabular}{|c|c|c|}
\hline Variable & $\mathrm{N}$ & n (\%) \\
\hline Experienced severe illness & 202 & $71(35.0)$ \\
\hline $\begin{array}{l}\text { Family members experienced severe } \\
\text { illness }\end{array}$ & 201 & $68(33.5)$ \\
\hline Duration of hypertension & 202 & \\
\hline$<6$ months & & $16(7.8)$ \\
\hline$\geq 6$ months & & $186(92.2)$ \\
\hline Last month blood pressure & 200 & \\
\hline$<140 / 90 \mathrm{mmHg}$ & & $110(54.2)$ \\
\hline$\geq 140 / 90 \mathrm{mmHg}$ & & $90(45.8)$ \\
\hline Health risk level ${ }^{\text {a }}$ & 186 & \\
\hline Low & & $51(25.1)$ \\
\hline Medium & & $100(49.3)$ \\
\hline High & & $26(12.8)$ \\
\hline Very high & & $9(4.4)$ \\
\hline Anti-hypertensive medications & 202 & \\
\hline 0 & & $16(7.9)$ \\
\hline 1 & & $134(66.0)$ \\
\hline 2 & & $44(21.7)$ \\
\hline$\geq 3$ & & $8(4.0)$ \\
\hline
\end{tabular}

mental health). The GenPHYS were measured by PF, RP, $\mathrm{BP}, \mathrm{RE}$, while the GenMENT were measured by $\mathrm{GH}, \mathrm{SF}$, $\mathrm{VT}, \mathrm{MH}$.

Figure 1 shows the measurement model resulted from EFA and substantial considerations. The ovals worded as GenPHYS and GenMENT represented latent variables. The rectangles represented the eight scales. The circles rightmost represented residual terms. The subscript numbers denoted measurement occasions $(1=$ baseline; $2=$ follow-up). The straight arrows represented loadings, and the curved arrows represented covariances. The residual factors were correlated across occasions. The residual factors for RP and RE were correlated by the instruction of the modification index, as both scales have close meaning and wording expression about social roles.

\section{Detection of $R S$ and adjusted change}

Details for model fit are given in Table 3, and Fig. 2 provided simplified patterns of the 4 longitudinal models used in RS detection.

Step 1: all fit indexes for model 1 were in an acceptable range, indicating an appropriate unconstrained measurement model was established.

Step 2: in model 2, all RS-related parameters were constrained to be invariant across occasions. The fit of model 2 was still acceptable but was significantly worse than model $1\left(\chi_{\text {SBdiff }}^{2}(20)=69.53, P<0.001\right)$, indicating the overall existence of RS. 


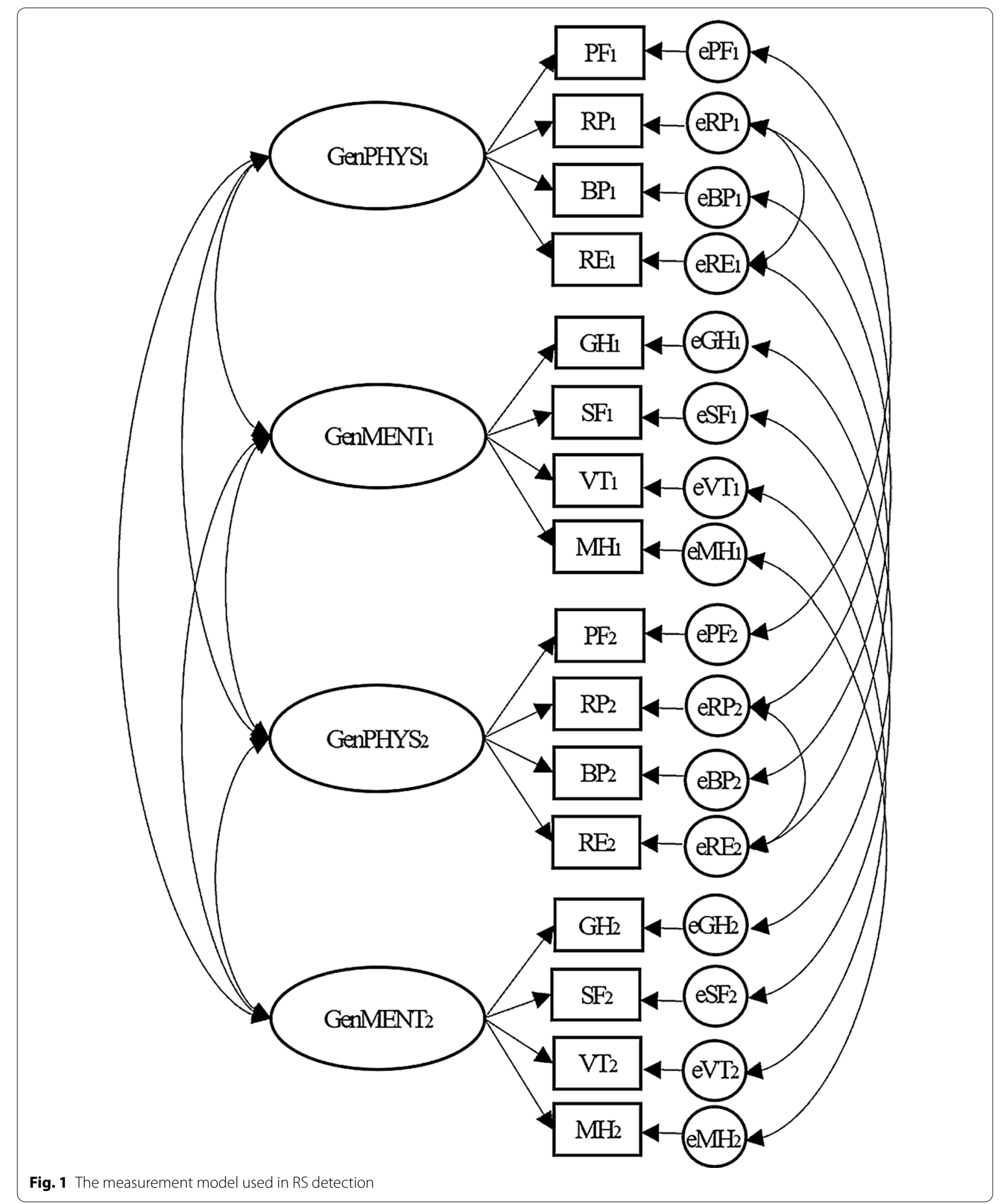


Table 3 Goodness of fit of models in the 4-step detection procedure

\begin{tabular}{lccllll}
\hline Model & Df & CHISQ & CFI & TLI & RMSEA (90\%CI) & SRMR \\
\hline Model 1 & 88 & 151.6 & 0.951 & 0.934 & $0.060(0.043,0.075)$ & 0.050 \\
Model 2 & 108 & 222.6 & 0.912 & 0.903 & $0.072(0.059,0.086)$ & 0.067 \\
Model 3 & 105 & 178.5 & 0.944 & 0.936 & $0.059(0.044,0.073)$ \\
Model 4 & 106 & 179.7 & 0.944 & 0.936 & $0.059(0.043,0.073)$ & 0.059 \\
\hline
\end{tabular}

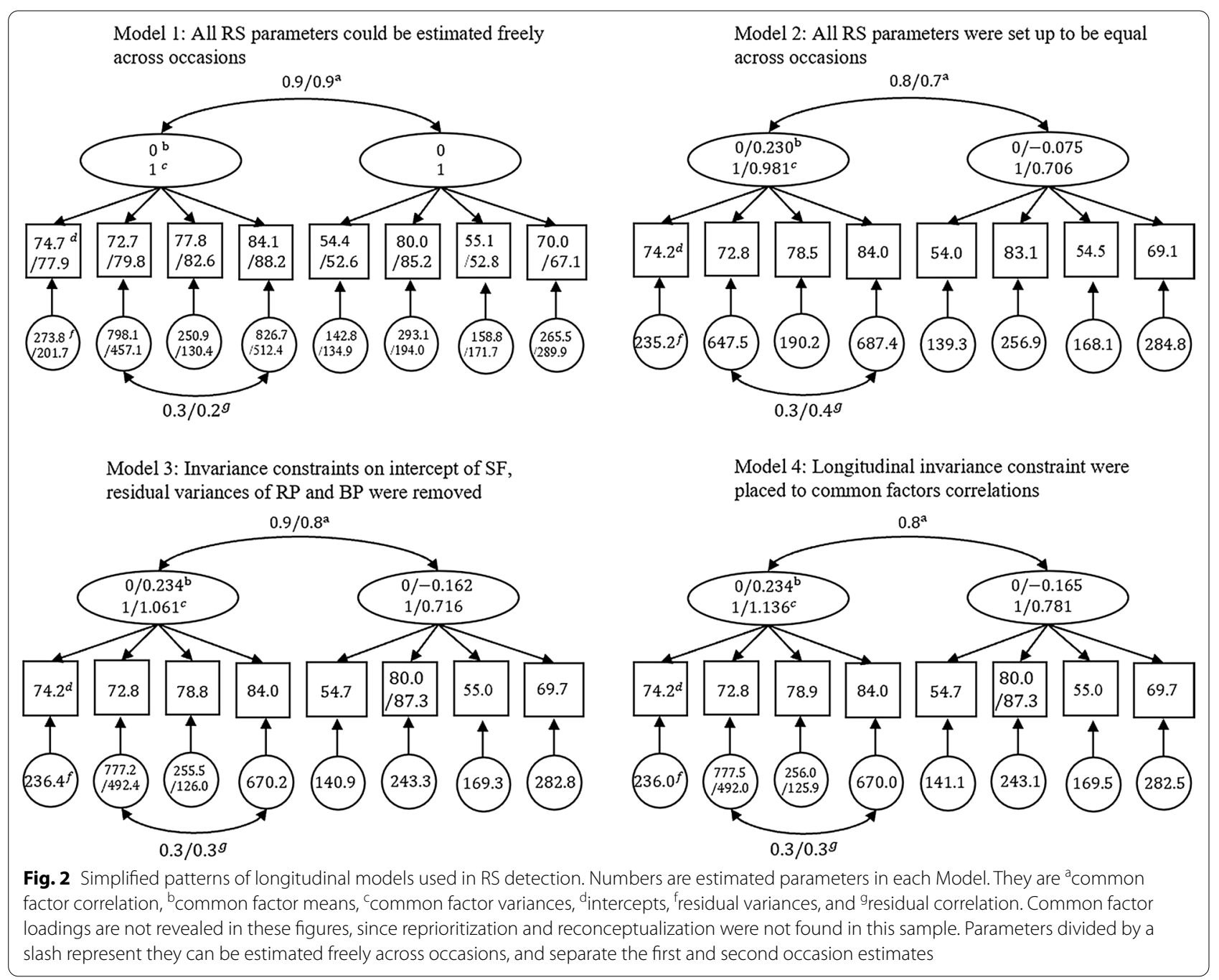

Step 3: after controlling for Type I error (Bonferroniadjusted critical value $=0.006)$, constrains of the residual variances of $\mathrm{RP}\left(\chi_{\mathrm{SBdiff}}^{2}(1)=8.84, \mathrm{P}=0.003\right)$ and $\mathrm{BP}$ $\left(\chi_{\text {SBdiff }}^{2}(1)=17.41, P<0.001\right)$, the intercept of SF $\left(\chi_{\text {SBdiff }}^{2}\right.$ $(1)=22.98, P<0.001)$ were removed, indicating non-uniform recalibration of $\mathrm{RP}$ and $\mathrm{BP}$, uniform recalibration of SF.

Step 4: the invariance constrain on common factor correlations across occasions were placed on model 4 . The longitudinal differences in both common factors means were significant, indicating a significant adjusted change in GenPHYS and GenMENT. The GenPHYS improved $(+0.234, \mathrm{P}<0.001)$, whereas the GenMENT deteriorated $(-0.165, \mathrm{P}=0.025)$, with effect-sizes all considered "small" (effect-sizes $=0.37,-0.21$ respectively). Estimated parameters of model 4 are presented in Table 4.

Table 5 shows a significant test of RS, and the effectsizes of observed change, RS, and adjusted change. RS in 
Table 4 Parameters estimated in model 4

\begin{tabular}{|c|c|c|c|c|c|c|c|c|}
\hline \multirow[b]{3}{*}{ Factor loading } & \multicolumn{4}{|l|}{ Pre-test } & \multicolumn{4}{|l|}{ Post-test } \\
\hline & \multicolumn{2}{|l|}{ GenPHYS $_{1}$} & \multicolumn{2}{|l|}{ Gen-MENT 1} & \multicolumn{2}{|l|}{$\mathrm{GenPHYS}_{2}$} & \multicolumn{2}{|l|}{ Gen-MENT 2} \\
\hline & & & & & & & & \\
\hline PF & 17.557 & & & & 17.557 & & & \\
\hline $\mathrm{RP}$ & 29.074 & & & & 29.074 & & & \\
\hline $\mathrm{BP}$ & 13.761 & & & & 13.761 & & & \\
\hline RE & 18.159 & & & & 18.159 & & & \\
\hline $\mathrm{GH}$ & & & 14.339 & & & & 14.339 & \\
\hline SF & & & 12.508 & & & & 12.508 & \\
\hline VT & & & 12.664 & & & & 12.664 & \\
\hline $\mathrm{MH}$ & & & 13.713 & & & & 13.713 & \\
\hline \multicolumn{9}{|l|}{ Intercepts } \\
\hline & PF & $\mathrm{RP}$ & $\mathrm{BP}$ & RE & $\mathrm{GH}$ & SF & VT & $\mathrm{MH}$ \\
\hline Pre-test & 74.214 & 72.804 & 78.853 & 83.962 & 54.656 & 80.049 & 55.038 & 69.664 \\
\hline Post-test & 74.214 & 72.804 & 78.853 & 83.962 & 54.656 & 87.290 & 55.038 & 69.664 \\
\hline \multicolumn{9}{|c|}{ Residual variance } \\
\hline & ResPF & ResRP & ResBP & ResRE & ResGH & ResSF & ResVT & ResMH \\
\hline Pre-test & 236.00 & 777.51 & 255.96 & 670.02 & 141.12 & 243.06 & 169.46 & 282.52 \\
\hline Post-test & 236.00 & 492.01 & 125.94 & 670.02 & 141.12 & 243.06 & 169.46 & 282.52 \\
\hline \multicolumn{9}{|c|}{ Common factor variances } \\
\hline & \multicolumn{3}{|l|}{ Pre-test } & & \multicolumn{2}{|l|}{ Post-test } & & \\
\hline & GenPHYS $_{1}$ & & Gen-MENT $_{1}$ & & $\mathrm{GenPHYS}_{2}$ & & \multicolumn{2}{|l|}{ Gen-MENT $_{2}$} \\
\hline & 1.00 & & 1.00 & & 1.136 & & 0.781 & \\
\hline \multicolumn{9}{|c|}{ Common factor correlations } \\
\hline \multicolumn{9}{|l|}{ Pre-test } \\
\hline GenPHYS $_{1}$ & \multicolumn{8}{|l|}{1} \\
\hline Gen-MENT $_{1}$ & \multicolumn{2}{|l|}{0.840} & \multicolumn{2}{|l|}{1} & & & & \\
\hline \multicolumn{9}{|l|}{ Post-test } \\
\hline $\mathrm{GenPHYS}_{2}$ & \multicolumn{2}{|l|}{0.871} & \multicolumn{2}{|l|}{0.685} & \multicolumn{2}{|l|}{1} & & \\
\hline Gen-MENT 2 & \multirow{2}{*}{\multicolumn{2}{|c|}{0.561}} & \multirow{2}{*}{\multicolumn{2}{|c|}{0.583}} & \multirow{2}{*}{\multicolumn{2}{|c|}{0.840}} & \multicolumn{2}{|l|}{1} \\
\hline \multicolumn{3}{|c|}{ Common factor means } & & & & & & \\
\hline & \multicolumn{4}{|l|}{ Pre-test } & Post-test & & & \\
\hline & GenPHYS & & Gen-MENT & & GenPHYS & & Gen-MENT & \\
\hline & 0.00 & & 0.00 & & $0.234 \mathrm{P}<0.001$ & & $-0.165 P=0.025$ & \\
\hline
\end{tabular}

Parameters of factor loadings are unstandardized; Results indicating across-measurement variance are printed in bold

the SF, RP, and BP scale were all significant. The effect was calculated as "small" for uniform recalibration of SF (effectsize $=0.35)$. RP and BP's effect-sizes were zero at the group level since the non-uniform recalibration indicated individual internal standard changes in different directions. After accounting for the RS effect, all scales except for the PF scale were stable. The PF scale of the participants improved slightly (effect-size $=0.21)$.

\section{Discussion}

This study explored the occurrence of RS in patients with hypertension attending the community disease management program by using Oort's SEM approach. In our sample, we detected indications of recalibration in the SF, $\mathrm{RP}$, and BP scale.

First, the intercept value of SF was higher at followup than baseline. This indicated that at follow-up, 
Table 5 Significance tests of RS, and the effect-sizes of observed change, RS, and adjusted change

\begin{tabular}{|c|c|c|c|c|c|c|}
\hline \multirow[t]{2}{*}{ Scale } & \multirow[t]{2}{*}{ RS } & \multicolumn{2}{|c|}{ Significance test } & \multicolumn{3}{|l|}{ Effect-sizes $^{a}$} \\
\hline & & $\chi_{\text {SBdiff }}^{2}(d f=1)$ & Prob & Observed change & RS & Adjusted change \\
\hline PF & & & & 0.21 & & 0.21 \\
\hline $\mathrm{RP}$ & Non-unif. recalibration & 8.84 & 0.003 & 0.19 & 0.00 & 0.19 \\
\hline $\mathrm{BP}$ & Non-unif. recalibration & 17.41 & $<0.001$ & 0.16 & 0.00 & 0.16 \\
\hline RE & & & & 0.12 & & 0.13 \\
\hline $\mathrm{GH}$ & & & & -0.13 & & -0.13 \\
\hline SF & Uniform recalibration & 22.98 & $<0.001$ & 0.25 & 0.35 & -0.10 \\
\hline $\mathrm{VT}$ & & & & -0.12 & & -0.11 \\
\hline $\mathrm{MH}$ & & & & -0.10 & & -0.10 \\
\hline
\end{tabular}

a Effect-sizes were calculated as corresponding parameters difference in model 4 divided by the estimated standard deviation; Values of 0.2, 0.5, 0.8 indicate "small", "medium", and "large" effect-sizes. Values less than 0.2 are considered "negligible";

Bonferroni-adjusted critical value $=0.006$

participants rated a better response category (higher scores) to social functioning than would be expected on account of the deterioration of their general mental health. The meaning of their response scale anchors of the SF scale may have been changed after the 4-weeks disease management program experiences, and this could be attributed to (uniformly) recalibration. Possible explanations for the result could be that the health education and social comparisons caused patients to alter their standards of comparison [20], and the reserve-building activities reduced health worries and increase calm and peaceful appraisal [34].

Second, the residual variances of $\mathrm{RP}$ and $\mathrm{BP}$ were lower at 4-weeks after baseline. This means the participants were less heterogeneous in what is specific to the measurement of role limitations due to physical problems and bodily pain. Therefore, some portion of changes in the observed variance of RP and BP cannot be explained by changes in the variability of the common factor (i.e., the general physical health), and it could be attributed to the (non-uniform) recalibration of a portion of the RP and $\mathrm{BP}$ response scale [8].

Since the non-uniform recalibration was defined as internal standard changes in different directions, the calculated effects on group level were absolutely zero. Whereas the effects of uniform recalibration in the SF scale were calculated as "small," and the influence on the measurement results was noticeable, which emphasized the importance of consideration to RS in longitudinal HRQOL studies. After accounting for RS effects, we found a slight improvement in general physical health (GenPHYS) and a slight deterioration in general mental health (GenMENT).

Studies of RS in HRQOL research have grown steadily in more than two decades, while the arguments about what RS really is have never been stopped. Oort and colleagues [35] defined RS as a special case of bias both from measurement perspective and conceptual perspective, which must be accounted for to arrive at a "true change." Whereas Rapkin and Schwartz treated RS as one kind of different changes in QOL, which were caused by the change of the way individual appraise his/her quality of life [36]. Nevertheless, they all agreed that RS effects should be treated as meaningful and distinct information rather than errors. We believe the RS effects should be recognized as an important and effective consequence derived from the disease management program and shall differ from the direct result that the interventions improved or deteriorated QOL, but rather mediated or moderated by the changes in the way individuals think about their QOL.

Prior research has explored the mechanisms where RS is produced. Sprangers and Schwartz [1] have proposed several mechanisms, including social comparisons and coping strategies. Empirical evidence has suggested that social comparison acted as a mediator between life events and RS [37]. Patients who considered themselves better off than others tend to maintain their quality of life even with a worsening functioning [38]. Oort inferred that learning how to cope with illness could induced RS in patients' physical functioning [7]. By integrating the appraisal process into the theoretical model of RS, Rapkin and Schwartz proposed the concepts and methods for direct measurements of the individual psychological process involved in rating a QOL item [20], which provided a deep insight into how and when RS occurred.

The then-test method was used to detect RS for this cohort in our previous work [39]. The results deviated in the PF scale, where the then-test has found the recalibration. The reason could be that the then-test approach was 
susceptible to inaccurate recall [40], especially among elderly participants [41], while the SEM approach was immune to recall issues.

This study has some limitations that should be noted. Although the measurement model that we used fitted the data well with sufficient statistical power, we remain cautious that our analytic sample maybe not large enough to draw substantive conclusions about this specific population. Besides, our sample size maybe not large enough for further multiple group analyses to explore the possible predictors of response shift within the SEM framework. Second, the group level model-based methods identify RS in terms of the variances of model patterns or parameters, but RS may not the only reason that arises these differences. This explained why the direct measurement of individual coping mechanisms and appraisal process is necessary. As Rapkin and Schwartz [36] mentioned, linking these group-level statistical methods to appraisal is central to the (RS research) field, which will be explored in our future studies.

\section{Conclusions}

In summary, this study identified the recalibration RS among patients with hypertension over a 4-week period of a disease management program. The RS effects on measurement results were significant and could be considered an important and complementary outcome derived from the intervention. Further studies with larger samples are necessary, and the direct measurement of the mechanism and appraisal process is recommended.

\begin{abstract}
Abbreviations
RS: Response Shift; SEM: Structural Equation Modeling; HRQOL: Health-Related Quality of Life; CAD: Coronary Artery Disease; SF-36: 36-Item Short Form Health Survey; PF: Physical Functioning; RP: Role Limitations due to Physical Problems; BP: Bodily Pain; GH: General Health; VT: Vitality; SF: Social Functioning; RE: Role Limitations due to Emotional Problems; MH: Mental Health; SD: Standard Deviation; EFA: Exploratory Factor Analysis; MLR: Robust Maximum Likelihood Estimator; CFI: Comparative Fit Index; TLI:Tucker-Lewis Index; SRMR: Standardized Root Mean Square Residual; RMSEA: Root Mean Square Error of Approximation; GenPHYS: General Physical Health; GenMENT: General Mental Health.
\end{abstract}

\section{Supplementary Information}

The online version contains supplementary material available at https://doi. org/10.1186/s12955-021-01732-w.

Additional file 1: Figure S1. Theoretical model of RS.

Additional file 2: Table S1. The result of selection bias test at baseline.

\section{Acknowledgements}

We thank Dr. Carolyn E. Schwartz for helpful suggestions. We are grateful to Zhaohui community health service center for the cooperation in this study.

\section{Authors' contributions}

HC performed statistical analysis, interpreted the data. $H C$ and LZ wrote the first draft. HW, PL, and HC designed the study. HW, RZ, XL, DLP, and TCE contributed to the writing of the final version of manuscript. All authors read and approved the final manuscript.

\section{Funding}

This study was supported by the National Natural Science Foundation of China (Nos. 70603024, 71573226).

\section{Availability of data and materials}

The datasets used and/or analyzed during the current study are available from the corresponding author on reasonable request.

\section{Declarations}

\section{Ethics approval and consent to participate}

All patients provided written informed consent to participate in the study. The protocol was approved by the Ethics Committee of Zhejiang University School of Medicine.

\section{Consent for publication}

Not applicable.

\section{Competing interests}

The authors declare that they have no competing interests.

\section{Author details}

${ }^{1}$ Department of Social Medicine of School of Public Health, Zhejiang University School of Medicine, 866 Yuhangtang Road, Hangzhou 310058, Zhejiang, People's Republic of China. ${ }^{2}$ Department of Public Health, Hangzhou Medical College, 481 Binwen Road, Hangzhou 310051, Zhejiang, People's Republic of China. ${ }^{3}$ Department of Pharmacy of the First Affiliated Hospital, Zhejiang University School of Medicine, 79 Qingchun Road, Hangzhou 310003, Zhejiang, People's Republic of China. ${ }^{4}$ Department of Health Services, University of Washington, H670 Health Sciences Building, Box 357660, Seattle, WA 98195-7660, USA.

Received: 17 September 2020 Accepted: 4 March 2021

Published online: 17 March 2021

\section{References}

1. Sprangers MAG, Schwartz CE. Integrating response shift into healthrelated quality-of-life research: a theoretical model. Soc Sci Med. 1999:48:1507-15.

2. Schwartz CE, Sprangers MAG. Methodological approaches for assessing response shift in longitudinal health related quality-of-life research. Soc Sci Med. 1999;48:1531-48.

3. Ahmed S, Bourbeau J, Maltais F, Mansour A. The Oort structural equation modeling approach detected a response shift after a COPD self-management program not detected by the Schmitt technique. J Clin Epidemiol. 2009;62(11):1165-72.

4. Visser MRM, Smets EMA, Sprangers MAG, de Haes HJ. How response shift may affect the measurement of change in fatigue. J Pain Symptom Manage. 2000;20(1):12-8.

5. Wang $X, X u X, H a n H$, et al. Using structural equation modeling to detect response shift in quality of life in patients with Alzheimer's disease. Int Psychogeriatr. 2019;31(1):123-32.

6. Verdam MGE, Oort FJ, Visser MRM, Sprangers MAG. Response shift detection through then-test and structural equation modeling: decomposing observed change and testing tacit assumptions. NEJP. 2012;67:58-67.

7. Oort FJ. Using structural equation modeling to detect response shifts and true change. Qual Life Res. 2005;14(3):587-98.

8. Oort FJ, Visser MRM, Sprangers MAG. An application of structural equation modeling to detect response shifts and true change in quality of life data from cancer patients undergoing invasive surgery. Qual Life Res. 2005;14(3):599-609. 
9. Sajobi TT, Brahmbatt R, Lix LM, Zumbo BD, Sawatzky R. Scoping review of response shift methods: current reporting practices and recommendations. Qual Life Res. 2018;27(5):1133-46.

10. Arija V, Villalobos F, Pedret R, et al. Physical activity, cardiovascular health, quality of life and blood pressure control in hypertensive subjects: randomized clinical trial. Health Qual Life Outcomes. 2018;16(1):184.

11. Trevisol DJ, Moreira LB, Kerkhoff A, Fuchs SC, Fuchs FD. Health-related quality of life and hypertension: a systematic review and meta-analyses of observational studies. J Hypertens. 2011:29(2):179-88.

12. Alonso J, Ferrer M, Gandek B, et al. Health-related quality of life associated with chronic conditions in eight countries: results from the International Quality of Life Assessment (IQOLA) Project. Qual Life Res. 2004;13(2):283-98.

13. Arslantas $D$, Ayranci U, Unsal A, Tozun M. Prevalence of hypertension among individuals aged 50 years and over and its impact on health related quality of life in a semi-rural area of western Turkey. Chin Med J (Engl). 2008;121(16):1524-31.

14. Bardage C, Isacson D, Ring L, Bingefors K. A Swedish population-based study on the relationship between the SF-36 and health utilities to measure health in hypertension. Blood Press. 2003:12(4):203-10.

15. Bar-On D, Lazar A, Amir M. Quantitative assessment of response shift in QOL research. Soc Indic Res. 2000;49(1):37-49.

16. Gandhi PK, Ried LD, Huang IC, Kimberlin CL, Kauf TL. Assessment of response shift using two structural equation modeling techniques. Qual Life Res. 2013;22(3):461-71.

17. Joint Committee for Guideline Revision. 2018 Chinese guidelines for prevention and treatment of hypertension - a report of the revision committee of chinese guidelines for prevention and treatment of hypertension. J Geriatr Cardiol. 2019;16(3):182-241.

18. Lix LM, Chan EK, Sawatzky R, et al. Response shift and disease activity in inflammatory bowel disease. Qual Life Res. 2016;25(7):1751-60.

19. Osborne RH, Hawkins M, Sprangers MA. Change of perspective: a measurable and desired outcome of chronic disease self-management intervention programs that violates the premise of preintervention/ postintervention assessment. Arthritis Rheum. 2006:55(3):458-65.

20. Rapkin BD, Schwartz CE. Toward a theoretical model of quality-of-life appraisal: implications of findings from studies of response shift. Health Qual Life Outcomes. 2004:2:1-12.

21. LV YR, Jin KL, Zeng HY, Gao C, Miao YY, Wang HM. Effects of comorbidity on quality of life for patients with common chronic diseases in community health service. Chin J Hosp Adm. 2012;28(12):925-8.

22. Wang HM, Patrick DL, Edwards TC, Skalicky AM, Zeng HY, Gu WW. Validation of the EQ-5D in a general population sample in urban China. Qual Life Res. 2012;21(1):155-60.

23. Gao C, Guo AY, Liu PP, Miao YY, LV YR, Wang HM. Application of the Chronic Care Model in hypertension management in community health services. Chin J Hosp Adm. 2012;28(12):921-4.

24. Kong JX, Zhu L, Wang HM, Li Y, Guo AY, Gao C, Miao YY, Wang T, Lu XY, Zhu HH, Patrick DL. Effectiveness of the Chronic Care Model in Type 2 Diabetes Management in a Community Health Service Center in China: A Group Randomized Experimental Study. J Diabetes Res. 2019;2019:6516581.

25. Li L, Wang HM, Shen Y. Chinese SF-36 Health Survey: translation, cultural adaptation, validation, and normalisation. J Epidemiol Community Health. 2003:57(4):259-63.
26. Ware JE, Snow KK, Kosinski M, Gandek B. SF-36 Health Survey: Manual and Interpretation Guide. Boston: Nimrod Press; 1993.

27. Kline RB. Principles and practice of structural equation modeling. New York: The Guilford Press; 2016

28. Browne $M$, Cudeck R. Alternative ways of assessing model fit. Testing structural equation models. London: Sage Publications; 1993.

29. Barclay-Goddard R, Lix LM, Tate R, Weinberg L, Mayo NE. Health-related quality of life after stroke: does response shift occur in self-perceived physical function? Arch Phys Med Rehabil. 2011:92(11):1762-9.

30. King-Kallimanis BL, Oort FJ, Visser MRM, Sprangers MAG. Structural equation modeling of health-related quality-of-life data illustrates the measurement and conceptual perspectives on response shift. J Clin Epidemiol. 2009;62(11):1157-64.

31. Cohen J. Statistical power analysis for the behavioral sciences. 2nd ed. Hillsdale, NJ: Erlbaum; 1988

32. Finney S, DiStefano C. Non-normal and categorical data in structural equation modeling. In: Hancock G, Mueller R, editors. Structural equation modeling: a second course. Greenwich: Information Age Publishing; 2006.

33. Satorra A, Bentler PM. Ensuring positiveness of the scaled difference chisquare test statistic. Psychometrika. 2010;75(2):243-8.

34. Schwartz CE, Zhang J, Michael W, Eton DT, Rapkin BD. Reservebuilding activities attenuate treatment burden in chronic illness: the mediating role of appraisal and social support. Health Psychol Open. 2018;5(1):2055102918773440.

35. Oort FJ, Visser MR, Sprangers MA. Formal definitions of measurement bias and explanation bias clarify measurement and conceptual perspectives on response shift. J Clin Epidemiol. 2009;62(11):1126-37.

36. Rapkin BD, Schwartz CE. Advancing quality-of-life research by deepening our understanding of response shift: a unifying theory of appraisal. Qual Life Res. 2019:28(10):2623-30.

37. Gibbons FX. Social comparison as a mediator of response shift. Soc Sci Med. 1999:48(11):1517-30.

38. Hagedoorn M, Sneeuw KC, Aaronson NK. Changes in physical functioning and quality of life in patients with cancer: response shift and relative evaluation of one's condition. J Clin Epidemiol. 2002;55(2):176-83.

39. Wang HM, Liu PP, Patrick DL, Edwards TC, Skalicky AM. Response shift in quality of life measurement among patients with hypertension in a community in China. Qual Life Res. 2010;19(Suppl):61-2.

40. Nagl M, Farin E. Response shift in quality of life assessment in patients with chronic back pain and chronic ischaemic heart disease. Disabil Rehabil. 2012:34(8):671-80.

41. Ahmed S, Mayo NE, Wood-Dauphinee S, Hanley JA, Cohen SR. The structural equation modeling technique did not show a response shift, contrary to the results of the then test and the individualized approaches. J Clin Epidemiol. 2005:58(11):1125-33.

\section{Publisher's Note}

Springer Nature remains neutral with regard to jurisdictional claims in published maps and institutional affiliations.

Ready to submit your research? Choose BMC and benefit from

- fast, convenient online submission

- thorough peer review by experienced researchers in your field

- rapid publication on acceptance

- support for research data, including large and complex data types

- gold Open Access which fosters wider collaboration and increased citations

- maximum visibility for your research: over 100M website views per year

At $\mathrm{BMC}$, research is always in progress.

Learn more biomedcentral.com/submissions 